\title{
The battle against cancer stem cells in glioblastomas
}

Glioblastomas are the most
common and one of the most
aggressive forms of brain tumour.
The severity of the cancer within
glioblastomas, along with the
potential for spreading, are
driven by a population of cells
known as cancer stem cells.
Cancer stem cells are the beating
heart of a cancer. Dr Tapinos
and his research team at Brown
University, Rhode Island have
uncovered a specific modification
to RNA and some of the key
regulators that perpetuate the
ability of cancer stem cells to
reoccur and metastasise. Lck is
one of these regulators and has
been identified as an important
therapeutic target.
he era of targeted therapy
has been revolutionary to our
understanding of how cancers work and the best way to treat cancer withou effects. Many patients hold out hope for novel therapeutic approaches, particul ily for glioblastomas as the prognosis is poor for this aggressive tumour type. One novel approach has been identified by Dr Nikos Tapinos and his team at Brown University. The researchers are exploring the ways in which cancer stem cells transition into differentiated cells. Their work has identified signalling pathways and RNA modifications as key regulators of this process.

CANCER STEM CELLS It was previously thought that a tumour that is based

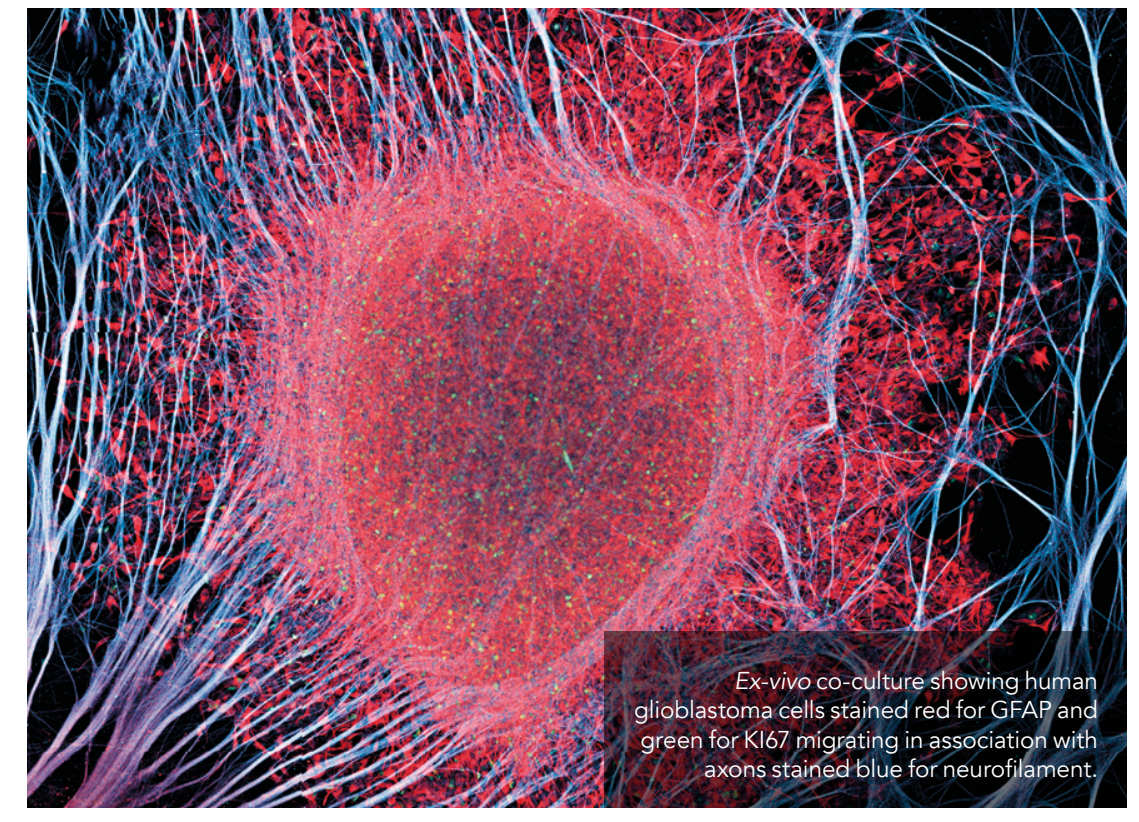

on the sub-type of cells and tissues from the part of the body it arose. Today, however, molecular technology has now know that more diverse cell types are often found within a malignant tumour One of the most central types of these cells is cancer stem cells; first discovered in leukaemia, stem cells have become an important area of research.

Gliomas are a type of tumour that develop in the brain and spinal cord, and make up $80 \%$ of all malignant brain tumours. There are different forms of gliomas such as astrocytoma, oligodendroglioma, and glioblastoma, however glioblastomas are one of the most fatal subtypes of not just gliomas, but of all tumours. This aggressive fumour is very difficult to treat despite surgical and medical interventions,

\section{CANCER STEM CELLS}

IN GLIOBLASTOMAS

Cancer stem cells are found in both

solid tumour and blood malignancies.

Although they have been implicated in contributing to the development of solid tumours, the exact nature of this process remains unclear. Just as norm stem cells can maintain the growth and replenishment of cells for organs, cancer stem cells drive the persistence of malignant tumour cells by producing new cancer cells.

Cancer stem cells operate similarly to normal tissue stem cells. Cancer stem cells can give rise to all cell types found a cancer sample. However, cancer stem
cells within glioblastomas have a high

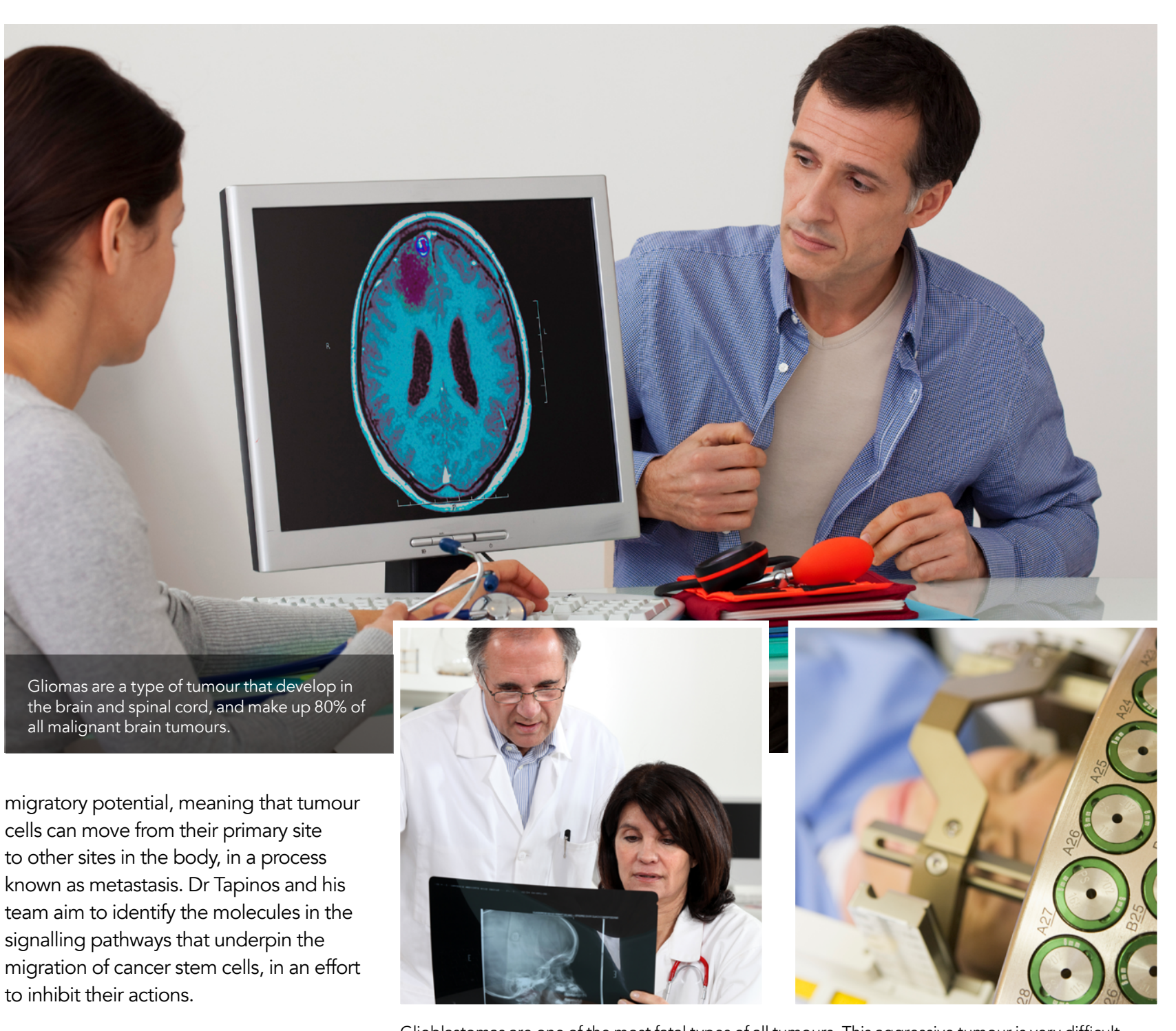

'Stemness' is an essential characteristic of stem cells that distinguish them from ordinary cells. It is the ability of that have been differentioned to have characteristics of a specific cell type The maintenance of tumour stemness glioblastomas have been linked to nonreceptor protein tyrosine kinases which regulate cellular processes.

Targeting cancer stem cells is key to fighting the battle against glioblastomas. As cancer stem cells are resistant to chemotherapy and radiation therapy, they contribute to the reoccurrence and of glioblastoma. The plasticity of the

Glioblastomas are one of the most fatal types of all tumours. This aggressive tumour is very difficult
to treat despite surgical and medical interventions, and reoccurrence is almost inevitable. cancer cells allows them to undergo various reversible transformations into the important structures that surround their surface This often means that cancer drugs that target the cells on the surface of the tumour may demonstrate limited efficacy.

LCK AS A THERAPEUTIC TARGET In recent years, protein tyrosine kinases have been shown to be key mediators in the development of malignancies. Indeed, Dr Tapinos and his colleagus showed that lymphocyte-specific

Importantly, this work demonstrates that Lck inhibitors could significantly reduce the possibility of the spread of glioma cells. regulators of glioma cell migration their work has cell identity. Importa significantly upregulated in glioblastor compared to the normal brain tissue, making Lck a good therapeutic target for targeting the malignant tissue as well as prevent cancer stem cell activity and spreading of glioma cells.

The Brown University team then tested an LCK-specific small molecule inhibitor on a glioblastoma animal model and found a significant reduction in the tumour area. The Lck inhibitor was also of most of the transcripts in cancer stem cells in glioblastoma. In addition, cancer cells have the abilty to form arm-like protrusions (called pseudopodia), which and therefore of cancer metastasis. shown to downregulate the expression 


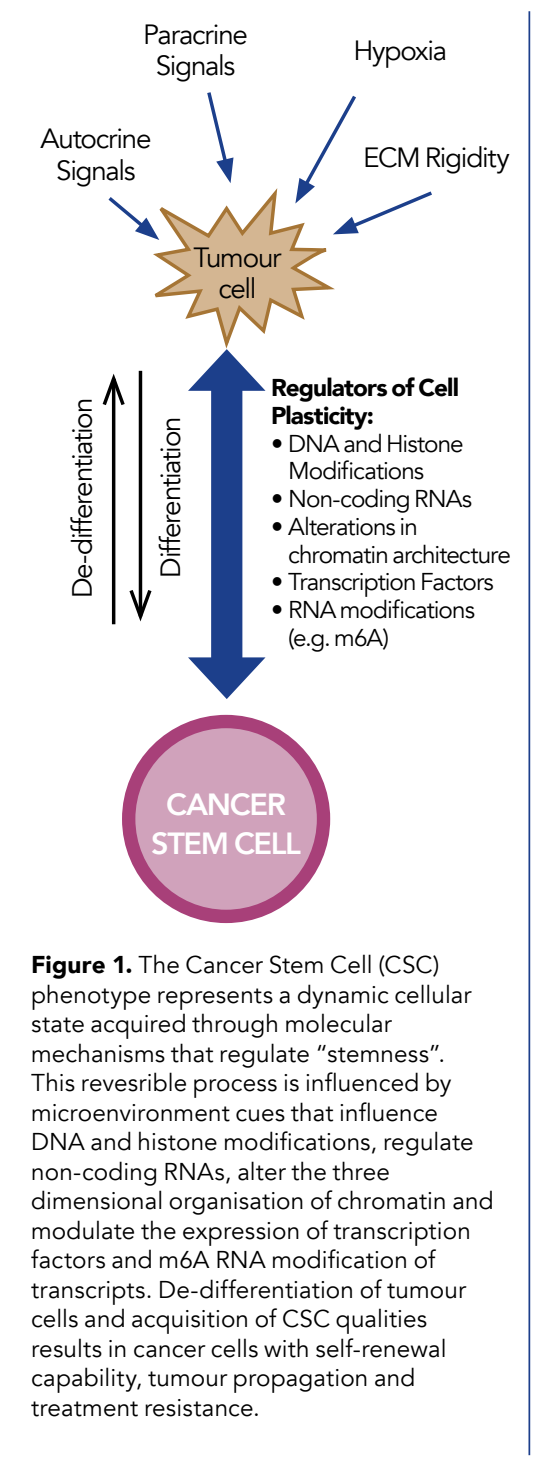

This work has the potential to change the outlook of patients all over the world who have been diagnosed with glioblastoma.

Interestingly, the Brown University researchers found that cells that were treated with Lck inhibitor formed fewer protrusions, therefore reducing the potential for the cancer spreading to secondary sites.

\section{M'A REGULATION}

Despite the importance of cancer

stem cell differentiation, one key are

that is poorly understood is the role of

epigenetic modification that regulates glioma cell function. This is where the comes in. N6-Methyladenosine ( $\left.{ }^{6} \mathrm{~A}\right)$ is a type of modification that occurs to RNA. While DNA provides the genetic information required for al living things, RNA, otherwise known as ribonucleic acid, is an important biological macromolecule that converts the genetic information of DNA into protein. $M^{6} A$ is a reversible modification that alters the way RNA behaves.

$M^{6} A$ can modify RNA using enzym writers and erasers called $\mathrm{m}^{6} \mathrm{~A}$ methyltransferases or demethylases respectively. $M^{6} A$ is the most prevalent internal modification for messenger RNAs, serving as a signal for translation of $\mathrm{mRNA}$ into protein or for regulation of mRNA stability and degradation. Dr Tapinos demonstrated that as glioblastoma cells transition from cancer stem cells to differentiated cells, they appear to lose the $\mathrm{m}^{6} \mathrm{~A}$ RNA methylation in certain genes and also show an increase in the rate of

\section{THE FUTURE}

In his future work, Dr Tapinos plans

to investigate how the tumour's

microenvironment regulates the post-

translational activity of RNA. He and specific RNAs could also be used to fine tune the cancer stem cell biologica modfications and constitute potentially new therapeutic or predictive targets in human glioblastomas. This work has the potential to change the outlook of been diagnosed with glioblastoma. his team have also demonstrated that patients all over the world who have
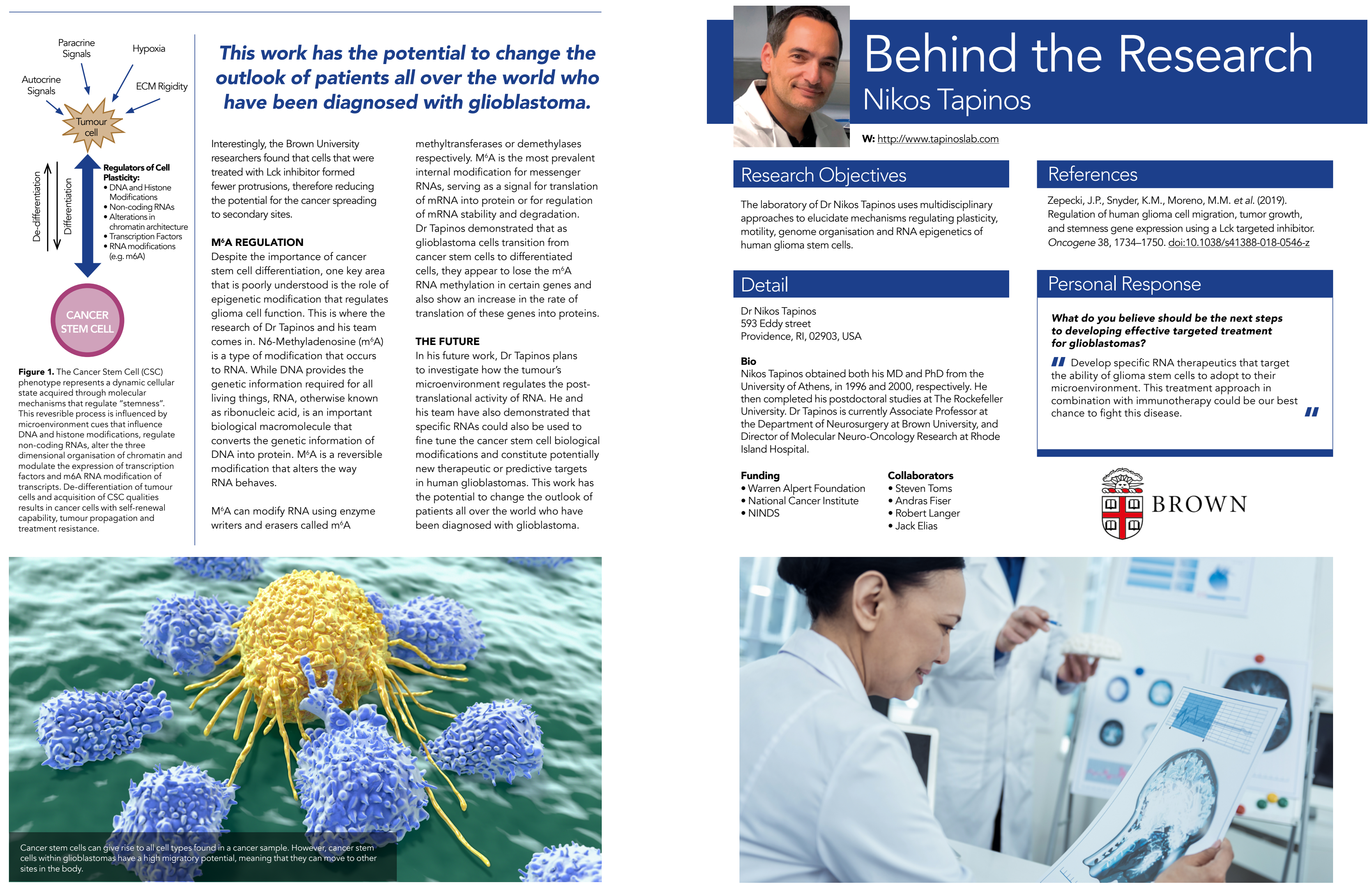

\section{Research Objectives}

The laboratory of Dr Nikos Tapinos uses multidisciplinary motility, genome organisation and RNA epigenetics of human glioma stem cells.

\section{Detail}

Dr Nikos Tapinos

Providence, RI, 02903, USA

Bio

Nikos Tapinos obtained both his MD and PhD from the University of Athens, in 1996 and 2000, respectively. He University. Dr Tapinos is currently Associate Professor at the Department of Neurosurgery at Brown University, and Director of Molecular Neuro-Oncology Research at Rhode

Funding

Collaborators - National Cancer Institute $\quad$ - Andras Fiser - NINDS $\quad$ • Anobert Langer - Jack Elias

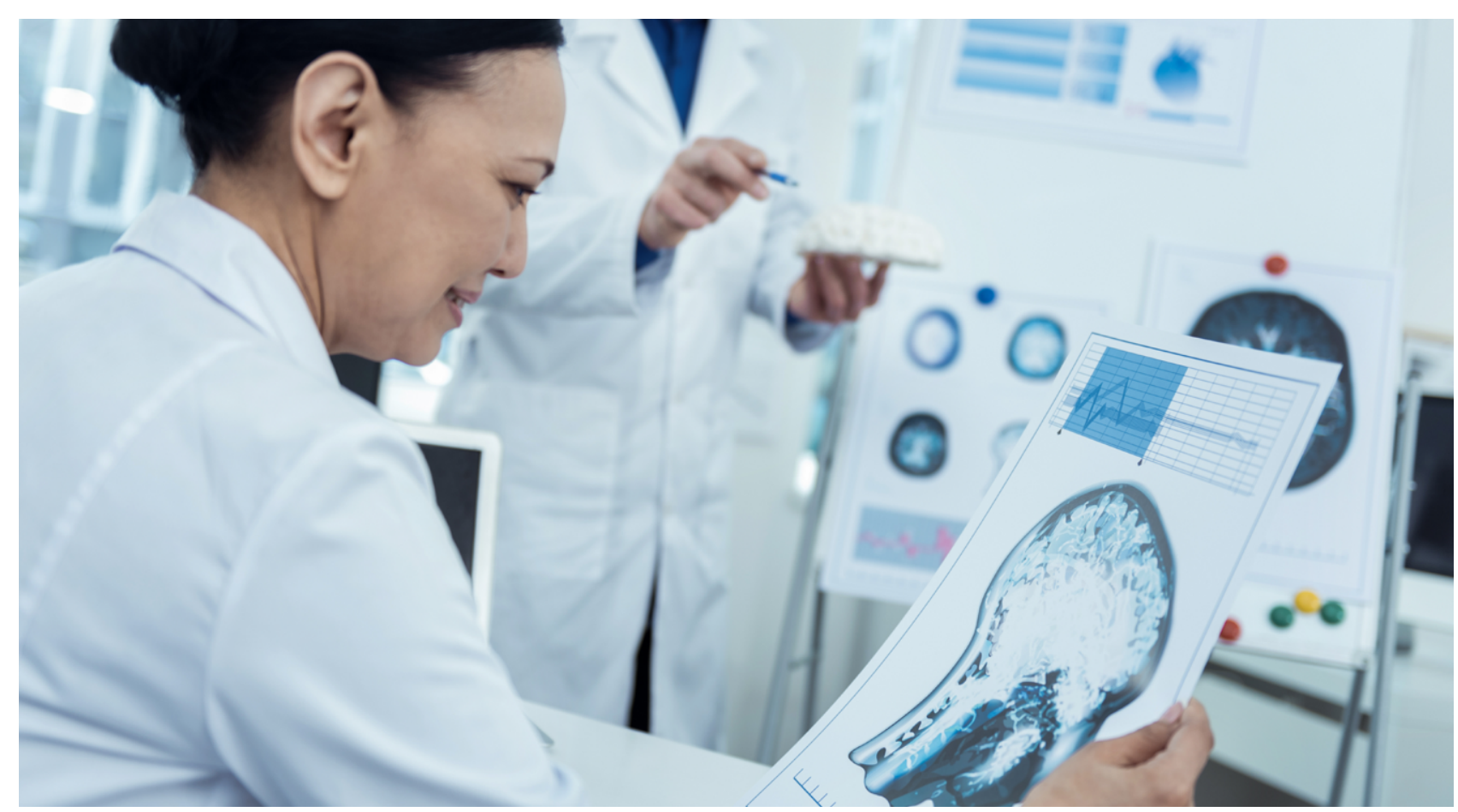

\section{References}

Zepecki, J.P., Snyder, K.M., Moreno, M.M. et al. (2019). Regulation of human glioma cell migration, tumor growth, and stemness gene expression using a Lck targeted inhibitor. Oncogene 38, 1734-1750. doi:10.1038/s41388-018-0546-z

\section{Personal Response}

What do you believe should be the next steps to developing effective targeted treatment

II Develop specific RNA therapeutics that target the ability of glioma stem cells to adopt to their

combination with immunotherapy could be our best

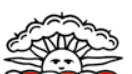

凹 $\mathrm{BROWN}$ 巴田 